\title{
Palmprint Recognition Based on Modular PCA and LS-SVM Li Kunlun ${ }^{1, a}$, Zhang Yaxin ${ }^{1, a}$, Liu Huanhuan ${ }^{1, a}$, Sun Shuo ${ }^{1, a,}$ \\ ${ }^{1}$ Electronic and Information Engineering, Hebei University, Baoding \\ axiwangzhikkkl@163.com
}

Keywords: Palmprint recognition; MPCA; LS-SVM classifier; single sample

\begin{abstract}
In this paper, in terms of feature extraction, according to the characteristics palmprint image we improve PCA algorithm and design modular PCA algorithms that is suitable for palmprint image. The whole palmprint image is divided into a plurality of sub-block images, then use principal component analysis. The improved feature extraction method is verified by the experiment and it can improve the recognition accuracy rate, especially in the case of single sample palmprint recognition. In the aspect of feature recognition, we train LS-SVM classifier to complete the palmprint recognition system.
\end{abstract}

\section{Introduction}

With the rapid development of network and communication technology, e-commerce worldwide is rising rapidly. Information security has become a key issue in today's information society development. Identification as an important means of information security, it is increasingly being taken seriously ${ }^{[1]}$. The traditional method is based on identification markers or knowledge. These methods have obvious problems: easily lost, forgotten or easily guessed passwords. Once the illegal user access to these identity markers, they can have the same powers of real users. The traditional identification of vulnerabilities caused huge loss.So people trying to seek more safe, reliable and popular authentication methods. Because palm is easily accessible and the main features are clear. Automatic palmprint recognition system has advantages of direct, friendly, unique and wide range of applications ${ }^{[2-3]}$.

Palmprint recognition based on principal component analysis is widely used method, and it is the main content of this research ${ }^{[4-5]}$. Because the principal component analysis is the overall sample were averaged, while retaining the main message of the original image will lose some image information $^{[6-7]}$. In this paper, palmprint recognition based on Modular PCA is without increasing the dimensions of the main ingredient. Especially in the case of single sample palmprint recognition, Modular PCA analysis accuracy increased significantly. Squares support vector machine is one of the methods improved of support vector machine. It is equality Constraints instead of the traditional support vector machine inequality constraints. Compared with support vector machine, least squares support vector machine in the loss of some precision on the basis of reducing the computational complexity and improve the model to solve the problem of speed. This article design MPCA method and least squares support vector machine combining palmprint recognition system and experiment verification.

\section{MPCA algorithm and least squares support vector machine}

\section{MPCA algorithm}

The traditional PCA method has its shortcomings. PCA is a linear transformation of the entire image in order to find an optimal set of orthonormal basis (that is the main component), with their linear combination to reconstruct the original sample. However, under normal conditions, a dimension palmprint image is very high, and image distribution is very compact, in such a high-dimensional space traditional PCA direct, very large computational complexity. The palm as a whole object feature extraction, will inevitably affect the relevance of global features, which fails to highlight some of the local in favor of classified information. To solve these problems, Vijayan K. Asari etc. proposed MPCA (Modular PCA) ${ }^{[8]}$.The purpose it is the training image divided into the 
corresponding number of sub-images, then all the sub-images use PCA to obtain the total covariance matrix.

MPCA algorithm:

Each image in the training set is divided into $P \times Q$ smaller images. For the training sample image $A_{i}$, it's $P \times Q$ sub-images can be represented mathematically as:

$$
A_{i}=\left(\begin{array}{cccc}
\left(A_{i}\right)_{11} & \left(A_{i}\right)_{12} & \cdots & \left(A_{i}\right)_{1 Q} \\
\left(A_{i}\right)_{21} & \left(A_{i}\right)_{22} & \cdots & \left(A_{i}\right)_{2 Q} \\
\vdots & \vdots & \ddots & \vdots \\
\left(A_{i}\right)_{P 1} & \left(A_{i}\right)_{P 1} & \cdots & \left(A_{i}\right)_{P Q}
\end{array}\right)
$$

where $\left(A_{i}\right)_{p q} \in R^{m_{1} \times n_{1}}, p=1,2, \cdots P, q=1,2, \cdots Q, m=m_{1} \times P, n=n_{1} \times Q$ 。

Similarly, the $P \times Q$ sub-images of the test sample image $I$ can be represented mathematically as

$$
I=\left(\begin{array}{cccc}
(I)_{11} & (I)_{12} & \cdots & (I)_{1 Q} \\
(I)_{21} & (I)_{22} & \cdots & (I)_{2 Q} \\
\vdots & \vdots & \ddots & \vdots \\
(I)_{P 1} & (I)_{P 1} & \cdots & (I)_{P Q}
\end{array}\right)
$$

According to this principle, the training set is divided into smaller images: $\left(\eta_{i}\right)_{p q}=\operatorname{Vec}\left(A_{i}\right)_{p q}$. Where $\left(\eta_{i}\right)_{p q} \in R^{\left(m_{1} \times n_{1}\right) \times 1}$, it's the column vector formed by the sub-picture. The overall covariance matrix of all the training sub-images is computed as:

$$
S=\frac{1}{M P Q} \sum_{i=1}^{M} \sum_{p=1}^{P} \sum_{q=1}^{Q}\left(\left(\eta_{i}\right)_{p q}-\eta\right)\left(\left(\eta_{i}\right)_{p q}-\eta\right)^{T}
$$

Where $\eta=\frac{1}{M P Q} \sum_{i=1}^{M} \sum_{p=1}^{P} \sum_{q=1}^{Q}\left(\eta_{i}\right)_{p q}$, it is the average image of all the training sub-images. Find optimal projection matrix $Z=\left[z_{1}, z_{2}, \cdots z_{r}\right]$, according to the overall covariance matrix $S$. Palmprint features can also be extracted according to the matrix $Z$.

$$
Y_{i}=\left(\begin{array}{cccc}
Z^{T}\left(\eta_{i}\right)_{11} & Z^{T}\left(\eta_{i}\right)_{12} & \cdots & Z^{T}\left(\eta_{i}\right)_{1 Q} \\
Z^{T}\left(\eta_{i}\right)_{21} & Z^{T}\left(\eta_{i}\right)_{22} & \cdots & Z^{T}\left(\eta_{i}\right)_{2 Q} \\
\vdots & \vdots & \ddots & \vdots \\
Z^{T}\left(\eta_{i}\right)_{P 1} & Z^{T}\left(\eta_{i}\right)_{P 1} & \cdots & Z^{T}\left(\eta_{i}\right)_{P Q}
\end{array}\right)=\left[\left(y_{i}\right)_{1},\left(y_{i}\right)_{2}, \cdots\left(y_{i}\right)_{Q}\right]
$$

$Y_{i}$ is the feature matrix of image $A_{i}$ that extracted based on MPCA algorithm, also known as the feature image, size $P_{r} \times Q$.

\section{LS-SVM}

Squares support vector machine is one of the methods improved of support vector machine. It is equality constraints instead of the traditional support vector machine inequality constraints. In this case the training loss of experience is the squared error and loss function, so that the support vector machine to solve the problem is transformed into a system of linear equations to solve the problem, shorten the time to solve. Compared with support vector machine, least squares support vector machine in the loss of some precision on the basis of reducing the computational complexity and improve the model to solve the problem of speed ${ }^{[9-11]}$.

Optimization SVM Vapnik proposed as follows:

$$
\left\{\begin{array}{l}
\operatorname{Min} J(\omega, \xi)=\frac{1}{2}\|\omega\|^{2}+C \sum_{i=1}^{N} \xi_{k} \\
\text { s.t. } y_{k}\left[\left(\phi\left(x_{k}\right) \omega^{T}+b\right)\right] \geq 1-\xi_{k}
\end{array}\right.
$$

Suykens proposed least squares support vector machine, optimizing the question becomes: 


$$
\left\{\begin{array}{l}
\operatorname{MinJ}(\omega, \xi)=\frac{1}{2}\|\omega\|^{2}+C \sum_{i=1}^{N} \xi_{k}^{2} \\
\text { s.t. } y_{k}=\phi\left(x_{k}\right) \omega^{T}+b+\xi_{k}
\end{array}\right.
$$

where, $\xi_{k} \geq 0, k=1,2, \cdots, N, C$ is a penalty factor 。

Solving optimization problems using Lagrange method (5),

$$
L(\omega, b, \xi, \alpha)=\frac{1}{2}\|\omega\|^{2}+C \sum_{i=1}^{N} \xi_{k}^{2}-\sum_{i=1}^{N} \alpha_{k}\left[\left(\omega^{T} \phi\left(x_{k}\right)+b+\xi_{k}\right)-y_{k}\right]
$$

Where $\alpha_{k}(k=1,2, \cdots, N)$ is lagrange multipliers.

According to the optimized conditions, the partial derivative of $\omega, b, \xi, \alpha$ and make it to zero.Kernel definitions $k\left(x_{i}, x_{j}\right)=\phi\left(x_{i}\right) \phi\left(x_{j}\right), k\left(x_{i}, x_{j}\right)$ is a symmetric function which satisfies Mercer condition. According to equation (7), the optimization problem is transformed into solving a linear equation:

$$
\left[\begin{array}{cccc}
0 & 1 & \cdots & 1 \\
1 & k\left(x_{1}, x_{1}\right)+1 / C & \cdots & k\left(x_{1}, x_{1}\right) \\
\vdots & \vdots & & \vdots \\
1 & k\left(x_{N}, x_{1}\right) & \cdots & k\left(x_{N}, x_{1}\right) 1 / C
\end{array}\right]\left[\begin{array}{c}
b \\
\alpha_{1} \\
\vdots \\
\alpha_{N}
\end{array}\right]=\left[\begin{array}{c}
0 \\
Y_{1} \\
\vdots \\
Y_{N}
\end{array}\right]
$$

Finally, get the nonlinear model is:

$$
f(x)=\sum_{k=1}^{N} \alpha_{k} k\left(x, x_{k}\right)+b
$$

\section{Palmprint recognition system based on MPCA algorithm and LS-SVM}

\section{Palmprint feature extraction algorithm based on MPCA}

In the study of palmprint recognition found when the palm rotation, illumination change to a certain extent, the recognition effect PCA is not so satisfactory. Inspired, the paper introduced the MPCA palmprint recognition, the palmprint image into many small sub-images and then these sub-images are identified using PCA. MPCA put palmprint image is divided into many sub-images, change the rotation and illumination changes only affect certain sub-image without affecting all sub-images, MPCA than traditional PCA achieved better recognition results.

Palmprint feature extraction algorithm based on the MPCA:

1、 Suppose there are $n$ test images, according to the formula (9) of each image is divided into $P \times Q$ smaller image .So each image $A_{i}$ into image $\Gamma_{i}$;

2、According to the formula (11) to calculate the total covariance matrix of all the training samples $S$;

3、 Next we find the eigenvectors of $S$ that are associated with the M0 largest $l$ eigenvalues $V_{i}(i=1, \cdots, l)$. Features palm (the training set) is $Y=\Gamma^{*} V$;
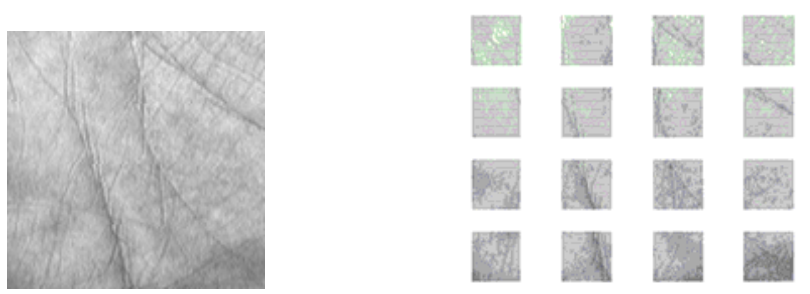

Fig.1.The sub-images of palmprint image

\section{Palmprint recognition system based on MPCA algorithm and LS-SVM}

With the new data set to train and LS-SVM classifier and classification verification, then get the recognition result. Fig. 2 shows the the specific process. 


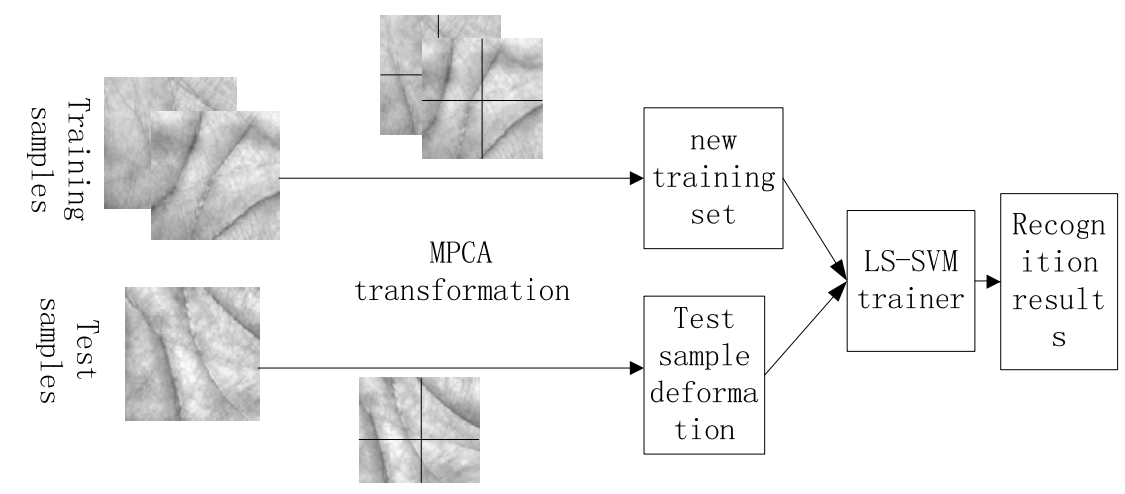

Fig.2.The sub-images of palmprint image

Identification process:

1、The first $i$ images of the test image set is $B_{i}$, which is divided into smaller image $\psi_{i}$.

2、 Complete the test image feature extraction by $Y T=\psi * V$ and then generate test set $Y T$.

3、 With training set $Y$ to train the LS-SVM trainer, to explore the optimal parameters;

Classify the test data with the classifier obtained in Step 6 and complete the identification.

\section{Experiment}

\section{Palmprint image preprocessing}

The database that we use in paper is The Hong Kong Polytechnic University (PolyU) Multispectral Palmprint Database. The database contains a total of 392 individual palmprint images were taken twice each 10 images, with a resolution of $384 * 284$. We first extract the 50 people 500 palmprint images were preprocessed images with a resolution of $128 * 128$. Pretreated paper includes two values, boundary tracking and key positioning step,the pre-processing palmprint image shown in Fig 6.

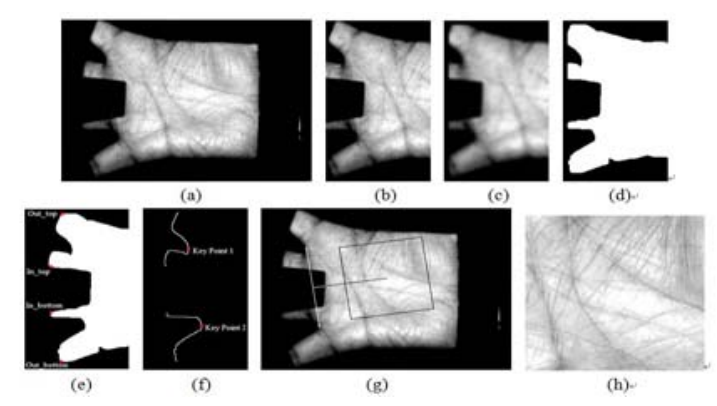

Fig.6. each pretreatment step (a)original image (b)image cropping (c)Gaussian smoothing(d) thres holding (e)the reference point detection (f)boundary tracking and critical point positioning(g)coordinate system is established(h)rotation and extracts the central region.

\section{Experiment}

Experiment 1: single sample palmprint recognition:

Single sample palmprint recognition is that use of only one image per palmprint database, through a certain algorithm analysis to complete the identification task from the palm database. Everyone's a palmprint as the sample is divided into blocks of $\mathrm{M} * \mathrm{~N}$. The remaining nine image as test images are classified according to the identified test procedure, the experimental results shown in Table 1. 
Table 1. Recognition rate when $\mathrm{m}, \mathrm{n}$ are different values

\begin{tabular}{|c|c|c|c|c|}
\hline n & 2 & 4 & 8 & 16 \\
\hline 2 & $52.4 \%$ & $64.2 \%$ & $60.2 \%$ & $50.2 \%$ \\
\hline 4 & $59.6 \%$ & $66.8 \%$ & $62.2 \%$ & $48.4 \%$ \\
\hline 8 & $63.4 \%$ & $63.8 \%$ & $58.6 \%$ & $48.2 \%$ \\
\hline 16 & $50.6 \%$ & $47.3 \%$ & $47.0 \%$ & $45.6 \%$ \\
\hline
\end{tabular}

As can be seen from the above table, different recognition results when different values of $\mathrm{m}$ and $\mathrm{n}$. When the number of partitions increasing, the recognition effect is gradually increasing. When the number of the sub-images to a certain extent, the recognition effect will be more low. The reason this phenomenon is associated with local features and global features of the image associated with it.

Take $\mathrm{m}, \mathrm{n}$ is 4,4 , We used the MPCA and PCA palmprint feature extraction, in the case of a single sample ,then combined with the LS-SVM classification and recognition. Take a different feature dimensions contrast test results shown in fig 4.

Derived from Figure 1, MPCA in a single sample palmprint recognition compared with PCA has the advantages of nearly 3 percent. This is because the reasonable sub-images, each one as a new sample. Each sub-image can be better preserved details compared to the overall PCA. With increasing characteristic dimension, recognition accuracy increased, but to a certain extent dimension, the accuracy rate stabilized.

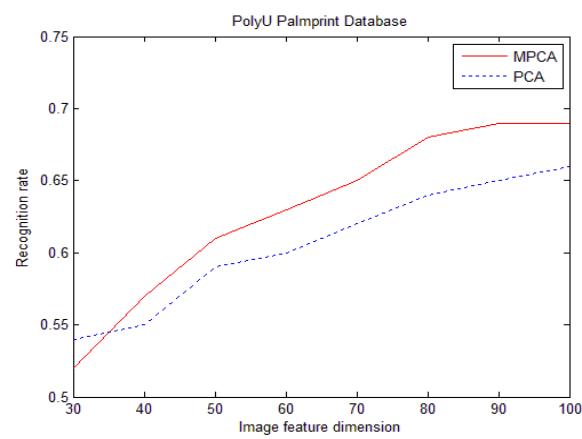

Fig.4. Single sample recognition rate

\section{Experiment 2: multiple categories experiment:}

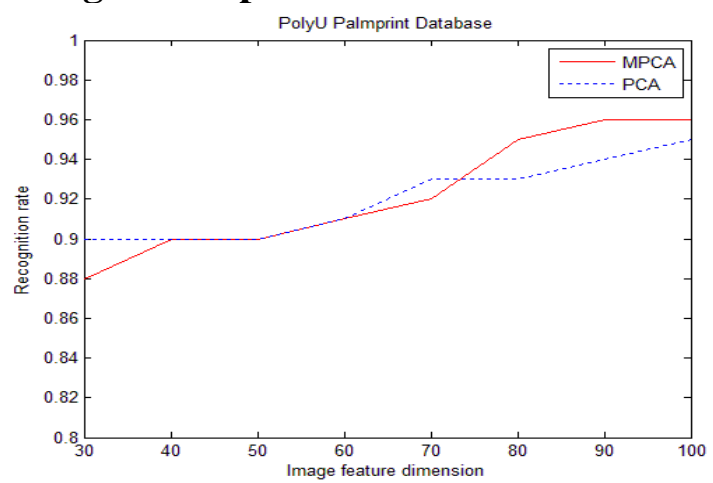

Fig.5. Multiple samples recognition rate

We put everyone's 3 images as the training set, and the remaining 7 images as the test set. Then repeat the experiment one, get experimental results shown in Table 2 and Figure 5 where $\mathrm{m}^{*} \mathrm{n}$ is $4 * 4$. 
Table 2. Recognition rate when $\mathrm{m}, \mathrm{n}$ are different values

\begin{tabular}{ccccc}
\hline & $\mathrm{m}$ & 2 & 4 & 8 \\
\hline 2 & & & \\
\hline & $89.4 \%$ & $93.8 \%$ & $91.4 \%$ \\
4 & $91.6 \%$ & $96.4 \%$ & $92.2 \%$ \\
8 & $89.8 \%$ & $93.2 \%$ & $90.4 \%$ \\
\hline
\end{tabular}

We can get from Table 2, when the training sample multiple, classification accuracy increase significantly and classification results are more credible. This is due to the classification based on more fully, more stable differences between classes when the training sample multiple. Figure 2 shows MPCA by nearly 1 percent accuracy rate compared with PCA palmprint recognition of this diversity, no single specimen obvious advantages.

\section{Summary}

We verify the feasibility of the MPCA through experiments. In recognition, we train LS-SVM classifier then design palmprint recognition system that combines MPCA and LS-SVM. Experiments show that when large palm database, it can avoid the small sample size problem and can reduce the amount of calculation. After the study, we can consider Weighted MPCA then verify it in theoretical and experimental aspects.

\section{Acknowledgement}

Thank the Hong Kong Polytechnic University for the palmprint database. This article is supported by the Graduate innovative projects of Hebei Province.

\section{Reference:}

[1] JAIN, A. K., ROSS, A, AND PRABHAKAR, S. An introduction to biometric recognition. IEEE Trans. Circuits Syst. Video Technol. 2004.14, 1, 4-20.

[2] Guo Z, Zhang D, Zhang L, et al. Feature band selection for online multispectral palmprint recognition[J]. Information Forensics and Security, IEEE Transactions on, 2012, 7(3): 1094-1099.

[3] Xu X, Zhang X, Lu L, et al. Fast near-infrared palmprint recognition using nonnegative matrix factorization extreme learning machine[J]. Optica Applicata, 2014, 44(2).

[4] Zhang D, Zuo W, Yue F. A comparative study of palmprint recognition algorithms. ACM Computing Surveys (CSUR), 2012, 44(1): 2.

[5] Nirosha Joshitha J, Selin R M. Image fusion using PCA in multifeature based palmprint recognition[J]. International Journal of Soft Computing and Engineering, 2012: P2.

[6] Guo J, Chen H, Li Y. Palmprint Recognition Based on Local Fisher Discriminant Analysis[J]. Journal of Software, 2014, 9(2): 287-292.

[7] Huang J, Su K, Elden J, et al. An MPCA/LDA Based Dimensionality Reduction Algorithm for Face Recognition[J]. Mathematical Problems in Engineering, 2014, 2014. 